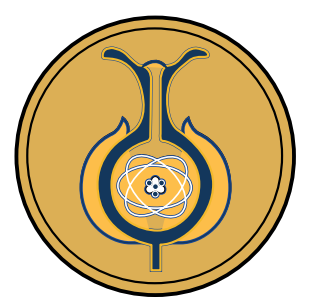

\title{
Optimización del cultivo larvario para la producción de juveniles del pargo manchado Lutjanus guttatus en Costa Rica
}

\author{
Optimization of larval culture for the production of juvenile spotted snapper \\ Lutjanus guttatus in Costa Rica
Otimização do cultivo larval para a produção de juvenis do Luciano Lutjanus guttatus na Costa Rica

\section{Jonathan Chacón-Guzmán ${ }^{1}$ Milagro Carvajal-Oses ${ }^{1}$ Ángel Herrera-Ulloa}

Received: Jul/1/2020 • Accepted: Nov/24/2020 • Published: Jul/31/2021

\section{Resumen}

La base tecnológica para la acuicultura del Lutjanus guttatus fue desarrollada en Costa Rica desde el 2002 por el Parque Marino del Pacífico. Las primeras producciones en masa de juveniles se obtuvieron en el 2005, las granjas comerciales a pequeña escala se establecieron a partir del 2006 y la primera transferencia de tecnología al sector privado inició en el 2008. A partir de estos avances se identificó la necesidad de optimizar la producción de juveniles. Con este objetivo, se evaluaron y describieron biológicamente tres cultivos larvarios obtenidos de desoves naturales de reproductores de segunda generación (F2). Tres familias $n=8$ cada una; $1,45 \pm 0,21 \mathrm{~kg} ; 1,33 \pm 0,12 \mathrm{~kg} ;$ y 1,28 $\pm 0,10 \mathrm{~kg}$ provenientes de una granja acuícola se aclimataron en el laboratorio hasta que desovaron. Se incubaron 100 mil huevos por desove (densidad: 520 huevos $\mathrm{mL}^{-1}$ ). Las larvas (90 mil/tanque) fueron sembradas con una longitud total (Lt) inicial de 2,25 mm y alimentadas con rotíferos enriquecidos, microalgas producidas en fotobiorreactores helicoidales y nauplios de artemia. El crecimiento, sobrevivencia, canibalismo, variabilidad del tamaño y deformidades presentadas fueron registradas. Un total de 22837 juveniles fueron cosechados en el día 60 después del desove. Durante la cosecha se obtuvo una longitud total del juvenil (Lt) de 57,8 \pm 7,82 $\mathrm{mm}$, tasa de crecimiento absoluto (TCA) 0,93 $\mathrm{mm} \mathrm{d}^{-1}$, tasa de crecimiento específico (TCE) $5,41 \% \mathrm{~d}^{-1}$, factor de condición (K) 1,089; una sobrevivencia promedio de 8,4 \% y 6,3\% de deformidades totales a la vista. Las metodologías utilizadas para obtener un desove natural de calidad, la mejora de alimento vivo producido por fotobiorreactores y la separación por tamaño fueron aspectos efectivos para el cultivo larval de la especie, aunque la inanición en los primeros días de alimentación exógena, la metamorfosis y el canibalismo siguen siendo factores que afectan la sobrevivencia final.

Palabras clave: Parque Marino; acuacultura; desove natural; producción de juveniles de peces.

Jonathan Chacón -Guzmán, $\bigotimes$ jonathan.chacon.guzman@una.cr, (1) https://orcid.org/0000-0002-8449-2334

Milagro Carvajal-Oses, $\nabla$ milagro.carvajal.oses@una.cr, (D) https://orcid.org/0000-0002-8294-9863

Ángel Herrera-Ulloa, $\$ angel.herrera.ulloa@una.cr, (D) https://orcid.org/0000-0003-2375-2945

1 Programa Parque Marino del Pacífico, Escuela Ciencias Biológicas, Universidad Nacional, Heredia, Costa Rica. 


\section{Abstract}

The technological foundation of the Lutjanus guttatus aquaculture was developed in Costa Rica in 2002 by Parque Marino del Pacífico. The first juvenile mass production was obtained in 2005; small-scale commercial farms were established in 2006; and the first technology transfer to the private sector began in 2008. Based on these advances, the need to optimize the production of juveniles was identified. With this objective, three larval crops obtained from natural spawning of second-generation (F2) breeders were evaluated and biologically described. Three families $n=8$ each: $1.45 \pm 0.21 \mathrm{~kg}, 1.33 \pm 0.12 \mathrm{~kg}$, and $1.28 \pm 0.10 \mathrm{~kg}$ from an aquaculture farm were acclimatized in the laboratory until they spawned. A total of $100,000 \mathrm{eggs}$ were incubated per spawning (density: 520 eggs $\mathrm{mL}^{-1}$ ). Larvae (90 thousand/tank) were seeded with an initial total length (TL) equal to $2.25 \mathrm{~mm}$ and fed with enriched rotifers, microalgae produced in helical photobioreactors, and artemia nauplii. Growth, survival, cannibalism, size variability, and deformities obtained were registered. A total of 22,837 juveniles were harvested on day 60 after spawning. During harvest, the juvenile's total length (TL) was $57.8 \pm 7.82 \mathrm{~mm}$, Absolute Growth Rate (AGR) was $0.93 \mathrm{~mm} \mathrm{~d}^{-1}$, Specific Growth Rate (SGR) was $5.41 \% \mathrm{~d}^{-1}$, Condition Factor (K) was 1.089 , average survival was $8.4 \%$ and total eye deformities were $6.3 \%$. Methodologies used to obtain natural quality spawning, improvement of live food produced by photobioreactors, and separation by size were effective aspects for the larval culture of the species, although starvation in the first days of exogenous feeding, metamorphosis, and cannibalism continue to be factors affecting final survival.

Keywords: Marine Park; aquaculture; natural spawning; production of juvenile fish

\section{Resumo}

A base tecnológica para a aquicultura do Lutjanus guttatus foi desenvolvida na Costa Rica desde 2002 pelo Parque Marino do Pacífico. As primeiras produções em massa de juvenis do Luciano foram obtidas em 2005, os viveiros comerciais de pequena escala foram estabelecidos a partir de 2006 e a primeira transferência de tecnologia ao setor privado iniciou em 2008. A partir desses avanços, identificou-se a necessidade de otimizar a produção de juvenis. Com este objetivo, foram avaliados e descritos biologicamente três cultivos larvais obtidos de desovas naturais de reprodutores de segunda geração (F2). Três famílias $n=8$ cada; $1,45 \pm 0,21$ $\mathrm{kg} ; 1,33 \pm 0,12 \mathrm{~kg} ; \mathrm{e} 1,28 \pm 0,10 \mathrm{~kg}$, provenientes de um viveiro aquícola, foram aclimatadas no laboratório até a desova. Foram incubados 100 mil ovos por desova (densidade: 520 ovos $\mathrm{mL}^{-1}$ ). As larvas ( $90 \mathrm{mil} /$ tanque) foram plantadas com uma longitude total (Lt) inicial de $2,25 \mathrm{~mm}$ e alimentadas com rotíferos enriquecidos, microalgas produzidas em fotobiorreatores helicoidais e náuplios de artemia. Houve o registro do crescimento, da sobrevivência, do canibalismo, da variabilidade do tamanho e das deformidades apresentadas. Foram coletados no total 22837 juvenis no dia 60 depois da desova. Durante a coleta, obteve-se foram obtidos uma longitude total do juvenil (Lt) de 57,8 \pm 7,82 mm, taxa de crescimento absoluto (TCA) 0,93 $\mathrm{mm} \mathrm{d}^{-1}$, taxa de crescimento específico (TCE) 5,41\% $\mathrm{d}^{-1}$, fator de condição (K) 1,089; e uma sobrevivência média de 8,4\% e 6,3\% de deformidades totais da vista. As metodologias utilizadas para a obtenção de uma desova natural de qualidade, a melhora de alimento vivo produzido por fotobiorreatores e a separação por tamanho foram aspectos efetivos para o cultivo larval da espécie, ainda que a inanição nos primeiros dias de alimentação exógena, a metamorfose e o canibalismo sigam sendo fatores que afetam a sobrevivência final.

Palavras-chave: Parque Marinho; aquicultura; desova natural; produção de juvenis de peixes. 


\section{INTRODUCCIÓN}

El pargo manchado Lutjanus guttatus (Steindachner, 1869) es considerado uno de los principales candidatos para el desarrollo de la piscicultura marina comercial en la costa pacífica tropical y subtropical de América Latina, gracias a que se distribuye desde el Golfo de California hasta Perú y a que cuenta con un mercado de exportación hacia EE.UU (Chacón-Guzmán, Carvajal-Oses, Herrera-Ulloa \& Pauletto, 2019a; Chacón-Guzmán, Carvajal-Oses, Toledo-Agüero \& Flores-Gatica, 2020; Fischer et al.,1995; Herrera-Ulloa, Chacón-Guzmán, Zúñiga-Calero, Fajardo-Espinoza \& Jiménez-Montealegre, 2009).

Los estudios para determinar su biotecnología de cultivo se han desarrollado especialmente en Costa Rica y México, aunque otros países como Colombia y Panamá también han realizado investigaciones con el fin de mejorar su producción en cautiverio (Abdo-de-la-Parra, Rodríguez-Ibarra, Rodríguez-Montes de Oca, Velasco-Blanco e Ibarra-Castro, 2015). Los primeros estudios con juveniles salvajes demostraron su factibilidad de crecimiento y sobrevivencia en jaulas flotantes a finales de la década de los noventa (Gutiérrez-Vargas \& Durán-Delgado, 1998). Las investigaciones realizadas tanto con desoves inducidos con hormonas como espontáneos han permitido estudiar aspectos reproductivos de la especie (Abdode-la-Parra et al., 2015; Álvarez-Lajonchére et al., 2012; Ibarra-Castro \& Duncan, 2007).

El escalamiento productivo que condujo al establecimiento de la base tecnológica de producción del pargo manchado L. guttatus en Costa Rica fue desarrollado a partir del año 2002 por el Parque Marino del Pacífico (PMP). Las producciones de juveniles iniciaron entre 2005 y 2006 con 45000 mil juveniles en total, los que permitieron implementar la primera granja de cultivo comercial en jaulas flotantes de pequeña escala manejada por pescadores artesanales. En 2007 se liberaron 71000 juveniles en el Refugio de Vida Silvestre Isla San Lucas y a partir del 2008 se iniciaron los procesos de venta de juveniles (117 000 individuos) y transferencia de la tecnología base a la primera empresa privada de gran escala, quienes en los siguientes años construyeron su propio centro de producción de juveniles y realizaron exitosamente sus primeras exportaciones a los Estados Unidos (Chacón-Guzmán et al., 2019b, 2020; Herrera-Ulloa et al., 2009; Herrera-Ulloa, Chacón-Guzmán, Zúñiga-Calero, Fajardo-Espinoza \& Jiménez-Montealegre, 2010; Velarde et al., 2012). En 2018, Costa Rica registró una producción de pargo de 600 toneladas métricas (Instituto Costarricense de Pesca y Acuicultura \& Secretaría Ejecutiva de Planificación Sectorial Agropecuaria, 2019). El escalamiento productivo desarrollado tanto en Costa Rica como en México ha permitido implementar las investigaciones hacia la mejora de los procesos de cultivo en jaulas flotantes (Castillo-Vargasmachuca, Ponce-Pelafox, Rodríguez-Domínguez \& Aragón-Noriega, 2016; Hernández et al., 2014; Hernández et al., 2016; Martínez-Cordero, Sánchez-Zazueta \& Hernández, 2017; Mazón-Suástegui et al., 2016; Peña, Hernández, Ibarra-Castro \& Álvarez-González, 2017).

Sin embargo, la fase larvaria del cultivo de peces ha representado un cuello de botella tecnológico para las especies que se han deseado cultivar (Tovar-Ramírez et al., 2017). Por ello, es necesario mejorar continuamente las metodologías de producción de peces marinos tales como reproducción, nutrición, bioseguridad y el manejo general de los cultivos larvales. 
El PMP como centro para la investigación y desarrollo de biotecnologías de cultivo de especies marinas de Costa Rica ha mejorado a través de los años los procedimientos de producción de juveniles. En este contexto, este trabajo tuvo como objetivo evaluar la producción de juveniles de tres cultivos larvales de L. guttatus obtenidos de desoves naturales de reproductores F2.

\section{METODOLOGÍA}

La investigación se realizó en el Laboratorio de Acuicultura y Biotecnología Marina del Parque Marino del Pacifico (PMP), Puntarenas, Costa Rica.

\section{Desove, incubación y siembra}

Tres desoves se obtuvieron de forma natural y espontánea de tres familias de reproductores $\mathrm{F} 2 ; \mathrm{n}=8 \mathrm{c} / \mathrm{u} ; 1,45 \pm 0,21$; $1,33 \pm 0,12 ; \mathrm{y} 1,28 \pm 0,10 \mathrm{~kg}$ (peso promedio \pm D.E.). Los reproductores se seleccionaron de la granja de cultivo de la Asociación de Acuicultores de Paquera (ASAP), ubicada en el Golfo de Nicoya $\left(9^{\circ} 48^{\prime} \mathrm{N} 84^{\circ} 48^{\prime} \mathrm{O}\right)$, los peces se marcaron por medio de sistemas de identificación de radiofrecuencia y fueron aclimatados en salas bajo techo con luz tenue y natural (fotoperiodo natural $\approx 12$ horas luz/oscuridad). Luego de tres meses de aclimatación iniciaron los desoves naturales en tanques circulares de fibra de vidrio de $20 \mathrm{~m}^{3}$, provistos de un sistema de recirculación (doble UV-65 watts, fraccionador de espuma RK2, filtro biológico y filtro mecánico) con un recambio de 8,6 volúmenes/ día y una reposición diaria del $5 \%$ con agua nueva filtrada a $5 \mu \mathrm{m}$ e irradiada (UV-128 watts). Los reproductores se alimentaron entre las 3:00 y 5:00 p.m., cinco días a la semana con calamar (Illex argentinus 40
$\%$ ), sardina (Opisthonema libertate $40 \%$ ) y camarón (Protrachynemme precipua $20 \%$ ). La relación macho hembra en cada tanque fue $1: 1$.

Los desoves se presentaron entre las 03:00 y 05:00 a.m. Los huevos boyantes fertilizados fueron desviados a través de un tubo lateral y superficial a un tanque cosechador de 1501 con un tamiz central de drenaje de $500 \mu \mathrm{m}, 9$ horas después fueron colectados y contados cada uno en una probeta de vidrio graduada de 11 . Los huevos se contaron por el método de desplazamiento volumétrico, luego de introducirlos se esperaron cinco minutos para que los huevos flotantes se separaran de los no flotantes. Para determinar cuántos huevos flotantes había por $\mathrm{ml}$ se contabilizaron cinco muestras de un $\mathrm{ml}$. El total de huevos, flotantes y no flotantes, se determinó multiplicando la cantidad de huevos de cada $\mathrm{ml}$ por el total de $\mathrm{ml}$ obtenidos en la columna de la probeta.

De la columna de huevos flotantes se tomaron tres muestras $(\mathrm{n}=30)$ y se revisaron en el microscopio, para determinar el diámetro del huevo y su gota de aceite, así como, el porcentaje de viabilidad, definida en base a los criterios establecidos por Silva \& Castelló (2005).

Los huevos fueron incubados a una densidad promedio de 520 huevos $\mathrm{ml}^{-1}$ en tanques cónicos de 2501 con agua de mar filtrada a cinco $\mu \mathrm{m}$ e irradiada (UV-128 watts). El recambio de agua durante la incubación fue de $91 \mathrm{~min}^{-1}$, con aireación moderada. Las incubadoras contaron con un tamiz central de drenaje de $150 \mu \mathrm{m}$.

Entre las 17 y 19 h después del desove (hdd) los huevos eclosionaron, a una temperatura entre 27,8 y $28,0^{\circ} \mathrm{C}$. Una hora después se detuvo el recambio y la aireación y se procedió a mover el agua circularmente hasta formar un efecto vortex, los restos 
de corion y huevos no eclosionados se concentraron en el centro inferior del tanque de donde fueron extraídos por sifón.

Para determinar el porcentaje de eclosión, se tomaron cinco muestras de un litro con un beaker graduado, el contenido se vació sobre una malla de $100 \mu \mathrm{m}$ y se procedió a contar las larvas eclosionadas. La siembra de las larvas se realizó por gravedad utilizando un tubo de una pulgada de diámetro. Los tanques de cultivo larval con un volumen total de $7 \mathrm{~m}^{3}$ se ubicaron bajo una malla sombra (sarán) con una penetración de luz del $20 \%$, contaron con un fraccionador de espuma de superficie y cinco piedras difusoras para proveer la aireación. La altura de la columna del agua fue de $0,90 \mathrm{~m}$ y el volumen del agua se mantuvo constante en $5 \mathrm{~m}^{3}$, el recambio por flujo continuo aumentó paulatinamente con los días de cultivo (Figura 1).

\section{Levante larval}

Se utilizó una densidad de cultivo similar para los tres tanques, la cual correspondió a 90 mil larvas aproximadamente. La alimentación se basó en la aplicación de microalgas marinas Nannochloropsis oculata, rotíferos Brachionus plicatilis, nauplios de Artemia sp. y alimento formulado (Figura 1).

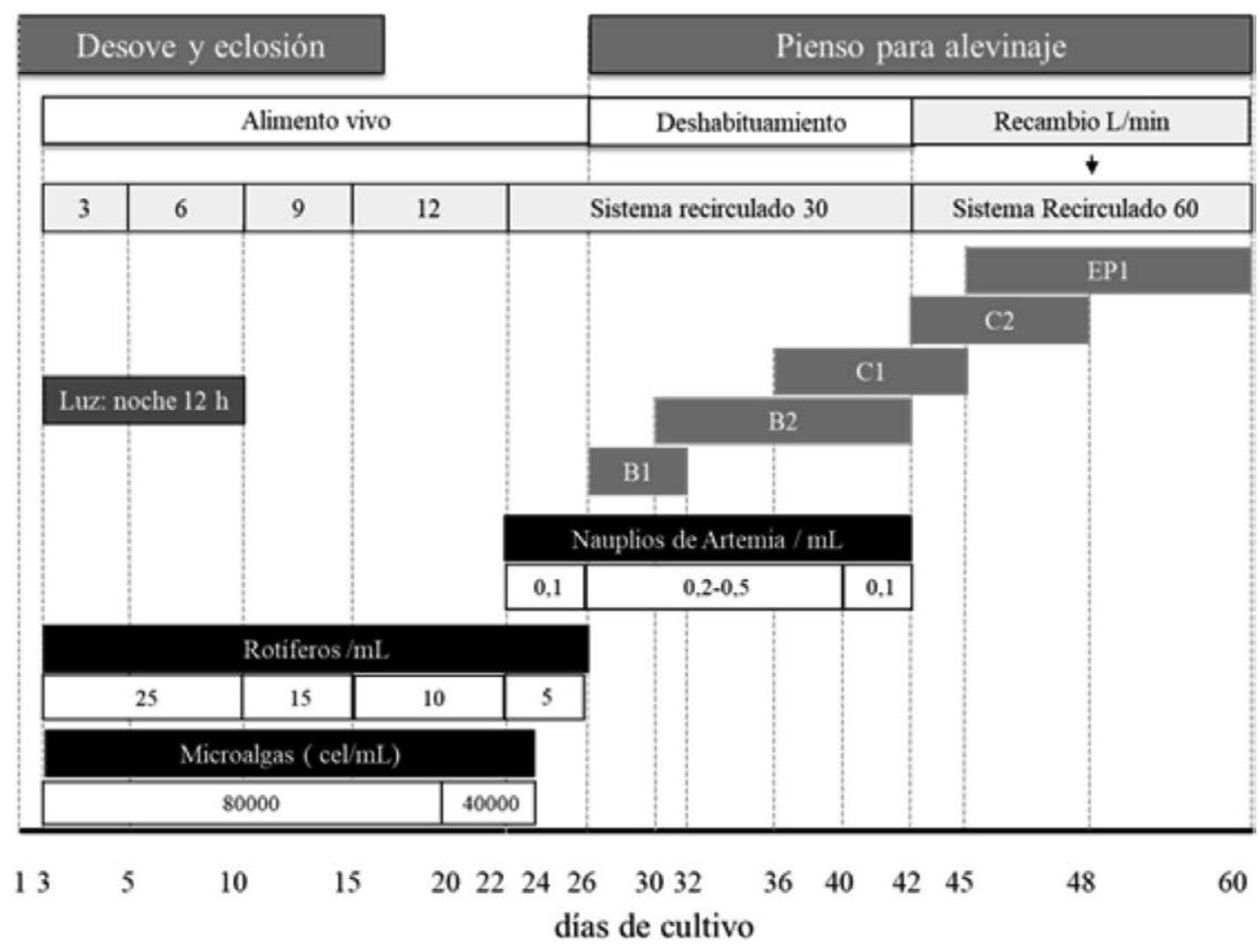

Figura 1. Alimentación, recambio de agua y luz artificial (día 3 al 10) aplicada en el proceso de producción de juveniles de L. gutattus en el Parque Marino del Pacifico. Donde

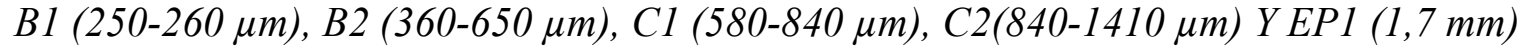
corresponde a tamaños del alimento formulado. Nota: Fuente propia de la investigación. 
Las microalgas fueron producidas por medio de un fotobiorreactor tubular helicoidal (Uribe \& Rangel, 2004), utilizando el medio nutritivo f/2 de (Guillard, 1975). Los medios de cultivo se mantuvieron a una salinidad de 31 PSU y fueron producidos en el cepario del laboratorio del PMP en el cual la temperatura se mantuvo en $27 \pm 1{ }^{\circ} \mathrm{C}$. Para facilitar el almacenaje en cámaras de refrigeración a $5{ }^{\circ} \mathrm{C}$, las microalgas fueron inducidas a la floculación por medio del protocolo de Rojo-Cebreros et al. (2016) y cuando fueron requeridas para su utilización, el alga floculada se vertió en envases con cinco litros de agua de mar y se procedió a la desfloculación por medio de la aplicación de dióxido de carbono durante 5 minutos a un flujo de $51 \mathrm{~min}^{-1}$.

Los rotíferos fueron producidos con un sistema intensivo basado en el protocolo de Carvajal-Oses, Campos-Rodríguez \& Herrera-Ulloa (2016). Los cultivos se mantuvieron a 31 PSU y a una temperatura promedio de $29,50 \pm 0,20{ }^{\circ} \mathrm{C}$. El enriquecimiento se realizó 12 horas antes de realizar la alimentación a las larvas y se basó en la fórmula utilizada por Benetti et al. (2008). Para entregar la cantidad necesaria de enriquecimiento a los rotíferos se utilizó la fórmula de Suantika, Dhert, Nurhudah \& Sorgeloos (2000):

$$
C H S=0,027 D^{0,415} \mathrm{~V}
$$

Donde:

$\mathrm{CHS}=$ total de alimento (gramos)

$\mathrm{D}=$ densidad de rotíferos $\mathrm{ml}^{-1}$

$\mathrm{V}=$ volumen

Los rotíferos se cosecharon en una malla de $50 \mu \mathrm{m}$, se lavaron con abundante agua de mar filtrada a $5 \mu \mathrm{m}$ e irradiada (UV-128 watts) y finalmente se mantuvieron en una hielera con temperatura aproximada de $10{ }^{\circ} \mathrm{C}$ para su posterior utilización como alimento.
La artemia se enriqueció durante $12 \mathrm{~h}$ en tanques cónicos de fibra de vidrio de 500 1. Al igual que con los rotíferos, para el enriquecimiento se utilizó el producto comercial DHA-Selco ${ }^{\circledR}$ de INVE Aquacuture, Bélgica. La dieta de enriquecimiento estuvo basada en la receta de Benetti et al. (2008); y fue entregada a la artemia a una tasa de 0,3 $\mathrm{g} / 10^{6}$ artemia. La artemia fue cosechada en una malla de $150 \mu \mathrm{m}$ y lavada durante 30 a 45 minutos con agua de mar filtrada a $5 \mu \mathrm{m}$ e irradiada. La artemia cosechada se almacenó en hieleras a una temperatura de $10{ }^{\circ} \mathrm{C}$ para su posterior entrega a las larvas.

La primera alimentación de las larvas al momento de la apertura de su boca, basada en microalgas y rotíferos, se ofreció a las 58 horas después del desove (hdd), antes de la pigmentación de los ojos. Durante el cultivo, entre 6:00-7:00 a.m. y 2:00-3:00 p.m., se aplicaron 5 litros de Nanochloropsis sp. $25 \times 10^{7}$ cel $\mathrm{ml}^{-1}$, junto con las microalgas se adicionaron, rotíferos Brachionus plicat ilis, disminuyendo progresivamente de 20 a 5 rotíferos $\mathrm{ml}^{-1}$ a lo largo del periodo, nauplios de artemia entre una y seis veces por día y entre $0,1 \mathrm{y}$ 0,5 nauplios $\mathrm{ml}^{-1}$. Para el deshabituamiento alimenticio se aplicó pienso en distintos calibres $(\leq 360 \mu \mathrm{m}, 360-650 \mu \mathrm{m}, 580-840 \mu \mathrm{m}$, $840-1,410 \mu \mathrm{m}$ y $1,5 \mathrm{~mm})$. Se aplicó entre 20 y 30 minutos antes de cada alimentación con nauplios de artemia (Figura 1).

Día por medio (un día sí y un día no) y durante el primer periodo de cultivo (26 días) se midió la longitud total (Lt) de las larvas $(n=15)$, inicialmente con un microscopio con lente micrómetro con escala de menor división de 25 micras y luego con una regla de $10 \mathrm{~cm}$ graduada en $\mathrm{mm}$. A partir del día $29 \mathrm{y}$ hasta el 60, además de obtener su Lt, las larvas se pesaron por medio de una balanza digital con una precisión de $\pm 0,01 \mathrm{~g}$. Se determinaron los siguientes índices de crecimiento: 
Tasa de crecimiento absoluto en peso:

$$
\text { TCA día }{ }^{-1}=\frac{(P f-P i)}{T}
$$

Tasa específica de crecimiento en peso:

$T E C(P C \%) d i ́ a{ }^{-1}=\frac{(\operatorname{Ln} P f-\operatorname{Ln} P i)}{T} * 100$

Tasa de crecimiento absoluto en longitud:

$T C A(L t m m) d i ́ a{ }^{-1}=\frac{(L f-L i)}{T}$

Tasa específica de crecimiento en longitud:

TEC $(L t \%) d i ́ a{ }^{-1}=\frac{(L n L f-L n L i)}{T} * 100$

Donde,

$\mathrm{Pf}=$ peso total promedio final $(\mathrm{g})$.

$\mathrm{Pi}=$ peso promedio inicial $(\mathrm{g})$.

$\mathrm{Lf}=$ longitud total promedio final $(\mathrm{mm})$.

$\mathrm{Li}=$ longitud promedio inicial $(\mathrm{mm})$.

$\mathrm{T}=$ número de días del período.

$\mathrm{Ln}=$ Logaritmo natural.

Con el peso final W y la longitud total final Lt se determinó el factor de condición (K), según Froese (2006):

$$
K=\frac{\mathrm{W}}{L T^{3}} * 100
$$

La sobrevivencia fue monitoreada en cinco diferentes etapas a partir del inicio del cultivo larval: a) a los tres días posteriores a la siembra; b) a los ocho días (fin de periodo de mayor mortalidad), c) a los 26 días (inicio del deshabituamiento alimenticio d) 42 días (final del deshabituamiento alimenticio) y e) a los 60 días (cosecha para traslado a las granjas de cultivo). Se bajó el nivel de agua de los tanques a 10001 , se aplicó $5 \mathrm{ml}$ de aceite de clavo de olor diluido [2-methoxy-4-2-(2-propenyl)phenol] para generar un efecto tranquilizante en las larvas y se procedió por medio de redes de mano (cachadores) a concentrarlas y contarlas en grupos de 100 ejemplares.
A los 17 días se inició la fase de separación de larvas para evitar el canibalismo. Del día 17 al 26 las larvas más grandes se capturaron con redes de mano y se trasladaron individualmente por medio de beakers de 2 litros de volumen a un tanque de $1,5 \mathrm{~m}^{3}$. En el día 27 todos los tanques se separaron en tres tallas (Small, Medium y Large). A partir de este momento los levantes larvales se unieron por talla en tres tanques de $10 \mathrm{~m}^{3}$ provistos con un sistema de recirculación (UV-65 watts, fraccionador de espuma, filtro biológico y filtro mecánico). Las larvas previamente separadas en el tanque de $1,5 \mathrm{~m}^{3}$ fueron adicionadas al tanque con larvas $\mathrm{L}$.

La cosecha de los juveniles de cada tanque se realizó a los 60 días. Los juveniles se anestesiaron con $15 \mathrm{ppm}$ de aceite de esencia de clavo de olor, se contaron uno a uno y se trasladaron en tanques transportadores de 5001 con $8 \mathrm{mg} \mathrm{l}^{-1}$ de oxígeno para el transporte hasta una granja de cultivo ubicada a 8,6 millas náuticas en el Golfo de Nicoya. Durante el conteo de cada tanque se tomó una muestra al azar de 300 juveniles, se anestesiaron con 20 ppm de aceite de clavo, se pesaron para determinar el peso promedio y se observaron detenidamente para determinar malformaciones físicas, el resultado se extrapoló a la población total.

Durante todo el periodo de producción se monitoreó el oxígeno disuelto (mg $\left.1^{-1}\right)$, la salinidad (PSU) y la temperatura $\left({ }^{\circ} \mathrm{C}\right)$.

Se analizó la normalidad y homocestacidad de los datos obtenidos. Se aplicó un análisis de varianza de una vía ANDEVA para determinar diferencias estadísticamente significativas entre las variables analizadas y se efectuaron con el programa Info Stat versión 2011 (Di-Rienzo et al., 2011). El crecimiento $(\mathrm{mm})$ hasta el día 26 , así como la $\mathrm{Lt}(\mathrm{mm})$ del día 26 al 60 de las larvas de cada cultivo fueron graficados en función del tiempo mediante hoja de cálculo Excel ${ }^{\circledR}$. 


\section{RESULTADOS}

\section{Desoves espontáneos naturales e incubación}

Con una salinidad de $30 \mathrm{PSU}$, temperatura de $28,9{ }^{\circ} \mathrm{C}$ y oxígeno disuelto de $6,1 \mathrm{mg}$ $1^{-1}$, se obtuvo un total de 514000 huevos provenientes de tres familias de reproductores F2.

No se presentaron diferencias significativas $(p \geq 0,05)$ entre la viabilidad y diámetro de los huevos de los tres desoves, ni entre los porcentajes de eclosión (Tabla 1).

\section{Cultivo larval}

Los parámetros físico químicos promedio obtenidos en el periodo de larvicultura y alevinaje fueron $6,67 \pm 0,71 \mathrm{mg} \mathrm{l}^{-1} \mathrm{de}$ oxígeno disuelto, $28,23 \pm 0,88^{\circ} \mathrm{C}$ de temperatura y $27,8 \pm 3,1 \mathrm{PSU}$ de salinidad.

La densidad de siembra fue similar entre los tres cultivos larvales, varió en 0,82 larvas $1^{-1}$ (rango entre 17,8 y 18,62), mientras que el promedio de la longitud total inicial de la larva fue de $2,25 \mathrm{~mm}$ sin diferencias significativas $(p \geq 0,05)$ entre los tres cultivos larvales (Tabla 1$)$.

Tabla 1. Características de los desoves y huevos, densidad de siembra y talla inicial de las larvas de L. guttatus

\begin{tabular}{lcccccccc}
\hline & \multicolumn{2}{c}{ Familia 1 } & \multicolumn{2}{c}{ Familia 2 } & \multicolumn{5}{c}{ Familia 3 } & $\begin{array}{c}\text { Promedio } \\
\text { general }\end{array}$ & $\boldsymbol{p}$ \\
\cline { 2 - 9 } & & $\mathbf{9}$ & & $\mathbf{\%}$ & & $\mathbf{\%}$ & $\mathbf{\%}$ & \\
\hline Desove & 198000 & & 145000 & & 171000 & & & \\
Huevos viables & 181000 & 91,4 & 134000 & 92,4 & 156000 & 91,2 & 91,7 & $\geq 0,05$ \\
Huevos malos & 17000 & 8,6 & 11000 & 7,6 & 15000 & 8,8 & 8,3 & \\
Huevos incubados & 100000 & & 100000 & & 100000 & & & \\
Eclosión & 89000 & 89 & 89500 & 89,5 & 93100 & 93,1 & 90,5 & $\geq 0,05$ \\
Diámetro del huevo $(\mu \mathrm{m})$ & $756,3 \pm 10,1$ & & $752 \pm 7,5$ & & $752 \pm 7,5$ & & & $\geq 0,05$ \\
Densidad de siembra (larvas $\left.1^{-1}\right)$ & 17,8 & & 17,9 & & 18,62 & & \\
Lt post eclosión $(\mathrm{mm})$ & $2,25 \pm 0,04$ & $2,25 \pm 0,03$ & & $2,25 \pm 0,04$ & & \\
\hline
\end{tabular}

Nota: Fuente propia de la investigación.

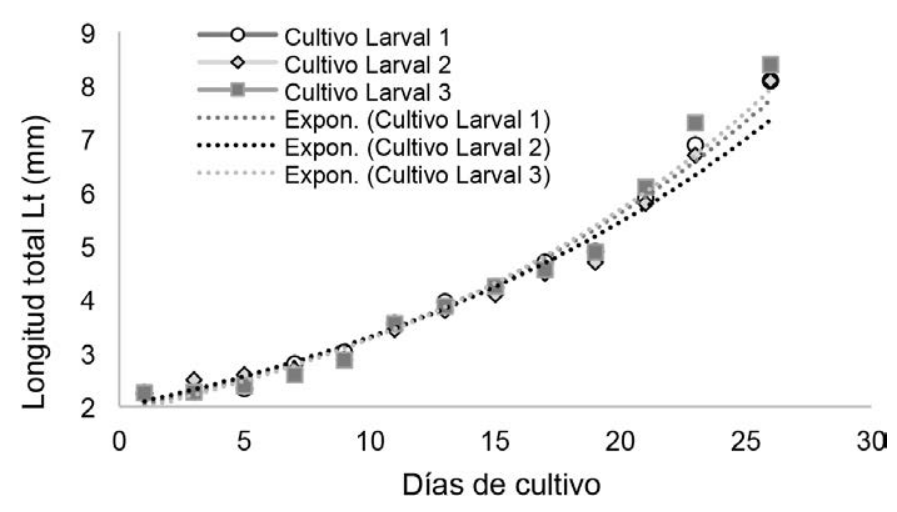

Figura 2. Crecimiento en longitud $(\mathrm{mm})$ de larvas de Lutjanus guttatus hasta los 26 dias del cultivo posteclosión.

Nota: Fuente propia de la investigación.
El crecimiento fue exponencial y presentó un comportamiento similar entre los tres cultivos larvales en la primera etapa del cultivo (26 días) (Figura 2). A los 26 días en promedio las larvas alcanzaron solamente $8,2 \pm 0,17$ $\mathrm{mm}$ de longitud total. La TCA (Lt) promedio alcanzado en este periodo fue de $0,23 \mathrm{~mm} \mathrm{~d}^{-1} \mathrm{y}$ la TCE (para Lt) de $4,97 \% \mathrm{~d}^{-1}$. No se presentaron diferencias significativas ( $p>0,05 \%$ ) entre el crecimiento (Lt) de los tres cultivos en los primeros 26 días. 
En el crecimiento de cada grupo de larvas, luego de su separación por tallas, al final del periodo se observó una leve tendencia a incrementar la diferencia de longitudes entre tallas separadas pasando de $8,61 \mathrm{~mm}$ a los 29 días a $15,10 \mathrm{~mm}$ a los 60 días (Figura 3).

El crecimiento promedio de las larvas durante el periodo de estudio fue exponencial (Figura 4). En promedio las larvas alcanzaron a los 60 días 57,8 \pm 7,82 mm de longitud total. La TCA (para Lt) promedio de todo el periodo fue de $0,93 \mathrm{~mm} \mathrm{~d}^{-1} \mathrm{y}$ la TCE de $5,41 \% \mathrm{~d}^{-1}$. El factor de condición $\mathrm{K}$ promedio obtenido al final del periodo de cultivo fue de 1,089.

La TCA en peso $(\mathrm{g})$ obtenida de cada grupo de larvas separadas por talla entre los 29 y 60 días fue: talla $(\mathrm{S})=0,05 \mathrm{~g}^{\text {día }}{ }^{-1}$; talla $(\mathrm{M})=$ $0,07 \mathrm{~g} \mathrm{día}^{-1}$; talla $(\mathrm{L})=0,08 \mathrm{~g} \mathrm{día}^{-1}$. La TCE: talla $(\mathrm{S})=17,3 \% \mathrm{~d}^{-1}$; talla $(\mathrm{M})=14,5 \% \mathrm{~d}^{-1}$; talla $(\mathrm{L})=12,0 \%$ $\mathrm{d}^{-1}$. La TCA y TCE en peso promedio fue de $0,06 \mathrm{~g} \mathrm{día}^{-1}$ y $16,9 \% \mathrm{~d}^{-1}$.

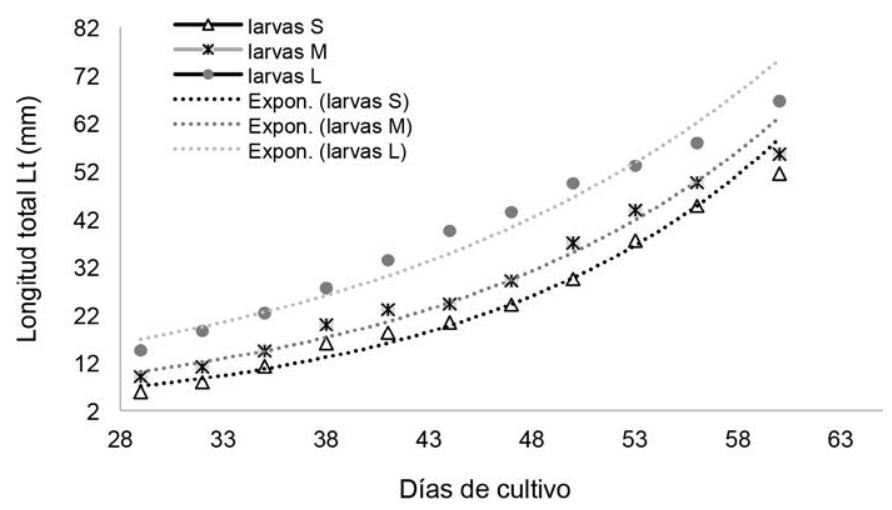

Figura 3. Crecimiento individual y promedio de las tres tallas $(S, M, L)$ obtenidas de la separación de los cultivos larvales de L. guttatus hasta la cosecha. Nota: Fuente propia de la investigación.

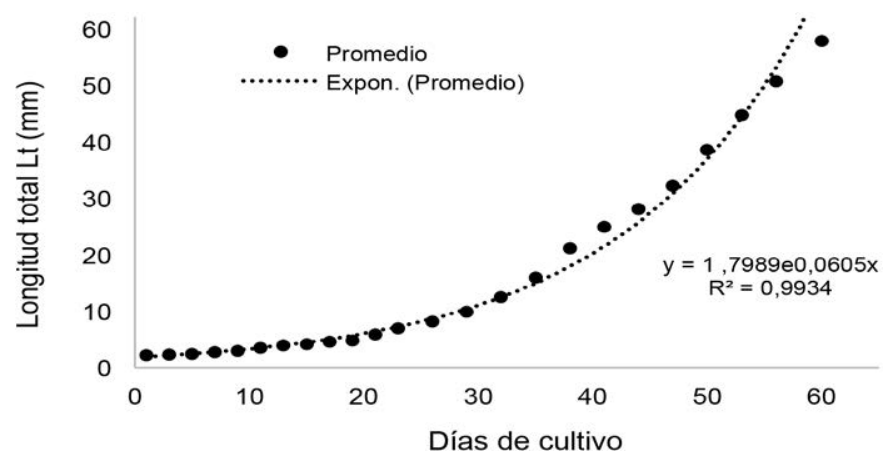

Figura 4. Crecimiento promedio de L. guttatus durante todo el periodo de cultivo. Nota: Fuente propia de la investigación.
La sobrevivencia fue monitoreada en dos etapas (Tabla 2), en la primera etapa (26 días) se da el mayor porcentaje de mortalidad promedio $88,1 \%$. En la segunda etapa (27-60 días) luego de la separación de tallas y cultivo en sistemas de recirculación

Tabla 2. Sobrevivencia monitoreada en el cultivo larval de L. guttatus antes y después de la separación por tallas

\begin{tabular}{|c|c|c|c|c|c|c|c|c|}
\hline \multirow[t]{2}{*}{ Día } & \multicolumn{2}{|r|}{ Familia 1} & \multicolumn{2}{|r|}{ Familia 2} & \multicolumn{2}{|r|}{ Familia 3} & \multirow{2}{*}{$\begin{array}{c}\text { Número larvas } \\
\text { total }\end{array}$} & \multirow{2}{*}{$\begin{array}{c}\text { \% Número larvas } \\
\text { total }\end{array}$} \\
\hline & $\%$ & Número de larvas & $\%$ & Número de larvas & $\%$ & Número de larvas & & \\
\hline $3 *$ & 100 & 89000 & 100 & 89500 & 100 & 93100 & 271600 & 100 \\
\hline 8 & 29 & 25810 & 25 & 22375 & 14 & 13034 & 61219 & 22,5 \\
\hline 26 & 17 & 15130 & 12 & 10740 & 7 & 6517 & 32387 & 11,9 \\
\hline \multirow[t]{2}{*}{ Día } & \multicolumn{2}{|c|}{ Tanque 4 (larvas S) } & \multicolumn{2}{|c|}{ Tanque 5 (larvas M) } & \multicolumn{2}{|c|}{ Tanque 6 (larvas L) } & Número larvas & \%Número larvas \\
\hline & $\%$ & Número de larvas & $\%$ & Número de larvas & $\%$ & Número de larvas & total & total \\
\hline 26 & 100 & $10040 * *$ & 100 & $14574 * *$ & 100 & $7773 * *$ & 32387 & 11,9 \\
\hline 42 & 74 & 7430 & 79 & 11513 & 93 & 7229 & 26171 & 9,6 \\
\hline 60 & 71 & 7128 & 75 & 8635 & 91 & 7073 & 22837 & 8,4 \\
\hline
\end{tabular}

*Día de la siembra

** Porcentaje de larvas separadas por talla a los 26 días (31 \% S, 45 \%, M y $24 \%$ L).

Nota: Fuente propia de la investigación. 
(RAS), la mortalidad bajó en comparación con la primera fase del cultivo, con una mortalidad de 3,5\%.

En la cosecha se determinó alta variabilidad de tallas; $31,2 \%$ de las larvas fueron pequeñas $(\mathrm{S}) \leq 1,4 \mathrm{~g} ; 37,8 \%$ presentaron una talla media (M) entre $\geq 1,5 \mathrm{~g} \mathrm{y} \leq 2 \mathrm{~g}$ y el $31 \%$ presentaron una talla grande $(\mathrm{L}) \geq 2,1 \mathrm{~g}$.

A los 60 días se presentó un 6,3\% de juveniles con algún tipo de deformidad observable como deformación del opérculo, mandíbula, lordosis y escoliosis (Tabla 3). La talla con mayor porcentaje de deformidad promedio fue la talla $\mathrm{S}$ con $8 \%$ y la de menor porcentaje la talla $\mathrm{L}$ con $5,3 \%$.

\section{DISCUSION}

La temperatura, salinidad y el oxígeno disuelto se mantuvieron estables y semejantes a las encontradas en la parte externa del Golfo de Nicoya (García-Rojas, Vega-Bolaños, \& Quesada-Phillips, 2017). Estos datos difieren a los reportadas por Álvarez-Lajonchére et al. (2012) para la producción larval de la misma especie; temperatura 26 $\pm 1,0{ }^{\circ} \mathrm{C}$; salinidad $35 \pm 1,0 \mathrm{~g} \mathrm{l}^{-1}$; oxígeno disuelto $5,1 \pm 0,6 \mathrm{mg} \mathrm{l}^{-1}$. En este trabajo las variables físico químicas fueron propicias para lograr desoves de buena calidad, según los estándares descritos por Silva \&
Castelló (2005) y adecuadas para los cultivos larvales y producción de juveniles.

Los reproductores de L. guttatus F2 alcanzaron puestas naturales luego de tres meses de su traslado de la granja a las instalaciones del PMP, un tiempo menor en comparación con los 12-13 meses descritos por Ibarra-Castro, García-Aguilar, Abdo de la Parra \& Rodríguez-Ibarra (2012) para alcanzar la madurez sexual con adultos salvajes de la misma especie. Los reproductores F2 seleccionados de granjas de cultivo se adaptan fácilmente a las condiciones de reproducción en cautiverio, lo cual contribuye a que el proceso de producción de huevos sea eficiente en términos de tiempo y economía.

La relación sexual fue efectiva para obtener puestas de buena calidad, a pesar de que para estos estudios generalmente se utiliza una relación sexual macho hembra de 2:1 (Abdo-de la Parra et al., 2015). La relación sexual 1:1 ha sido empleada en el PMP con el objetivo de lograr una mayor disponibilidad de ovocitos maduros por tanque en los periodos de alta demanda de juveniles para el cultivo en granjas marinas.

Valores altos en viabilidad y eclosión de los huevos, como los obtenidos en este trabajo, han sido considerados por Bromage et al. (1992) como factores de buena calidad del huevo. Gutiérrez-Sigeros, Ibarra- Castro

Tabla 3. Porcentajes por tipo de deformidad obtenidos de las muestras de cosecha de los juveniles de L. guttatus cultivados en el PMP

\begin{tabular}{|c|c|c|c|c|c|c|c|c|}
\hline & \multicolumn{2}{|c|}{$\mathbf{S}$} & \multicolumn{2}{|c|}{ M } & \multicolumn{2}{|c|}{$\mathbf{L}$} & & \\
\hline $\mathrm{N}$ & 7128 & & 8635 & & 7073 & & & \\
\hline$n$ & 300 & $\%$ & 300 & $\%$ & 300 & $\%$ & \# Promedio & $\%$ Promedio \\
\hline Opérculo & 18 & 6,0 & 16 & 5,3 & 14 & 4,7 & 16,0 & 5,3 \\
\hline Lordosis & 1 & 0,3 & 0 & 0,0 & 0 & 0,0 & 0,3 & 0,1 \\
\hline Escoliosis & 2 & 0,7 & 0 & 0,0 & 1 & 0,3 & 1,0 & 0,3 \\
\hline Boca & 3 & 1,0 & 1 & 0,3 & 1 & 0,3 & 1,7 & 0,6 \\
\hline Total & 24 & 8,0 & 17 & 5,7 & 16 & 5,3 & 19,0 & 6,3 \\
\hline
\end{tabular}

Nota: Fuente propia de la investigación. 
\& Álvarez-Lajonchére (2018), con Ocyurus chrysurus, obtuvieron adecuados desarrollos larvales con $87,3 \%$ de huevos flotantes de los cuales el $90 \%$ eran transparentes con embriones vivos.

El diámetro del huevo, la longitud inicial de la larva y el porcentaje de eclosión se encontraron dentro del rango de valores normales reportados para la especie por varias investigaciones (rango del diámetro del huevo 650 a $900 \mu \mathrm{m}$; longitud total entre 2,1 y $2,7 \mathrm{~mm}$; eclosión $\geq 80 \%$ ), mientras que el porcentaje de fecundación estuvo por encima del rango del $90 \%$ (Abdo-de-laParra et al., 2015).

En condiciones normales y con buena calidad de huevos se esperan eclosiones superiores al $80 \%$ (Silva \& Castelló, 2005). El promedio de eclosión superó este valor por $10,5 \%$. Respecto a otras especies del mismo género fue superior al reportado por Papanikos, Phelps, Ferry \& Maus (2003) para Lutjanus campechanus $(83,6 \pm 9,5 \%) \mathrm{y}$ por Hamamoto et al. (1992) para el Lutjanus stellatus ( $80 \%$ ) y para la misma especie superior a reportados por Herrera-Ulloa et al., (2009) (85\%) y similar al reportado por Álvarez-Lajonchère et al., (2012) (90,2 $\pm 2,1$ $\%$ ). La ausencia de diferencias estadísticamente significativas en viabilidad, diámetro de los huevos y porcentaje de eclosión demostró que las condiciones de cultivo y alimentación de reproductores fueron similares y propicias para los tres desoves y su respectiva incubación, lo que permitió continuar con la fase de cultivo larval.

La densidad de siembra empleada en el cultivo larval (17,8-18.62 larvas/ $\left./ l^{-1}\right)$ se mantuvo dentro del rango descrito por Abdo-de-la-Parra et al. (2010) como un rango de densidad que no afecta negativamente el crecimiento y la sobrevivencia larval de la especie.
La ausencia de diferencias significativas $(p \geq 0,05)$ entre los valores de crecimiento en los primeros 26 días demostró homogeneidad en las condiciones de cultivo y crecimiento en las larvas provenientes de los tres desoves.

Las talla promedio obtenida a los 26 días $(8,2 \mathrm{~mm})$ fue inferior a la reportada por Boza-Abarca, Calvo-Vargas, Solís-Ortíz \& Komen (2008) a los 25 días (10,23 $\mathrm{mm}$ ), pero similar a las descrita por Álvarez-Lajonchére et al. (2012) a los 25 días $(8 \mathrm{~mm})$. En este trabajo, el crecimiento larval se incrementó luego de la aplicación de los nauplios de artemia y en especial con la separación de tallas desde los 26 hasta los 60 días para alcanzar una talla levemente superior de $57,8 \mathrm{~mm}$ a la reportada para la misma especie por Álvarez-Lajonchére et al. (2012) (55 mm). A la misma conclusión llegaron García-Ortega et al. (2005), quienes también reportaron un aumento en el crecimiento de las larvas a partir de la alimentación con nauplios de artemia.

En comparación con otras investigaciones de la misma especie, durante los primeros 26 días, se presentaron leves diferencias en los valores de crecimiento. La TCA $\left(0,23 \mathrm{~mm}\right.$ día $\left.^{-1}\right)$, fue superior a la obtenida por Álvarez-Lajonchére et al. (2012) a los 25 días $\left(0,22 \mathrm{~mm}^{\prime}\right.$ día $\left.^{-1}\right)$ e inferior a la reportada por Boza-Abarca et al. (2008) $(0,243$ $\mathrm{mm}$ día $\left.^{-1}\right)$. El peso final promedio alcanzado a los 60 días $(1,90 \pm 0,90 \mathrm{~g})$, aunque inferior al reportado por Álvarez-Lajonchére et al. (2012) $(2,24 \pm 0,04 \mathrm{~g})$, se considera adecuado para iniciar el traslado a las granjas de cultivo. En comparación con otras especies de pargo, el crecimiento en la etapa larval en esta especie tiende a ser inferior (Emata, Eullaran \& Bagarinao, 1994; Ogle \& Lotz, 2011). El factor de condición fue inferior al reportado por Álvarez-Lajonchére et al. 
(2012) aunque se encuentra dentro del intervalo de los datos reportados para peces marinos (Moretti, Fernández-Criado \& Guidastri, 1999).

Un alto porcentaje de larvas durante los días 3-4 ddd no presentaron alimento en su sistema digestivo, lo que es considerado como principal causante del 77,5 \% de mortalidad medido a los ocho días de cultivo larval. En Colombia se registró $85 \%$ de mortalidad a los cinco días después de la eclosión y se asumió la pérdida por la misma situación (Sierra-de-la-Rosa, 2007). Una probable causa es que la boca del $L$. guttatus es muy pequeña para su presa $(0,105 \pm 0,010$ $\mathrm{mm}, \mathrm{n}=100$ ) (Boza-Abarca et al., 2008), ya que los rotíferos adultos de $B$. plicatilis cultivados en el PMP presentaron tallas promedio de $163,17 \pm 31,85 \mathrm{~mm}$ de longitud y $127,88 \pm 32,46 \mathrm{~mm}$ de ancho. Para minimizar el problema se optó por un sistema de cultivo de agua verde en los primeros días (Liao, Su \& Chang, 2001; Naas, Naes \& Harboe, 1992; Palmer, Burque, Palmer \& Burke, 2007). Este sistema permitió la reproducción de los rotíferos en el tanque de cultivo larval y, por ende, mayor disponibilidad de juveniles de rotíferos con tallas aproximadas a los $75 \mu \mathrm{m}$, lo que aumenta el porcentaje de captura e ingestión de las larvas.

Una de las principales causas de mortalidad, después del día 20, en este tipo de larvas se debe al canibalismo (Hecht \& Piennar, 1993; Ibarra-Castro, Gutiérrez-Sigeros, Álvarez-Lajonchére, Durryty-Lagunes \& Sánchez-Zamora, 2015). Se infiere que el segundo periodo de mortalidad monitoreado a los 26 días del cultivo larval (25\%) pudo estar relacionado en un alto porcentaje al canibalismo, el cual fue evidente a partir del día 14 y acentuado a partir del día 25; por lo que se optó por la separación de tallas a los 26 días. La separación de tallas al día 26 podría ser un factor que, además de influir en el crecimiento de las larvas de L. guttatus, contribuye en la disminución del canibalismo.

La diferenciación natural de tallas y la aplicación de una ración de alimento por debajo de la adecuada permiten el aumento en la frecuencia de ataques de canibalismo (Baras \& Jobling, 2002; Qin \& Fast, 1996), por ello, es necesaria la observación constante del cultivo larval de L. guttatus a partir del día 14-15 días, para identificar el inicio del canibalismo, así como un monitoreo constante del alimento sobrante en el tanque y el consumido por las larvas. En consecuencia con los sugerido por Rosenlund, Stoss \& Talbot (1997), se recomienda el suministro temprano del alimento artificial junto con el alimento vivo, por lo que es importante conocer cómo se desarrolla su sistema digestivo.

La variabilidad de tallas obtenida en la cosecha (sesenta días) fue similar a otras especies de peces marinos (Hecht \& Piennar, 1993; Moretti et al., 1999), y se considera al canibalismo un factor causal en la diferencias de tallas (Hecht \& Piennar, 1993).

Ambos aspectos explican la diferencia de tallas en esta especie. Silva \& Oliva (2010) reportaron que en el lenguado chileno Paralichthys adspersus, a los 60 días, un $67 \%$ de individuos alcanza tallas cercanas a los $15 \mathrm{~mm}$ y un $33 \%$ a los $20 \mathrm{~mm}$., mientras que Ibarra-Castro et al. (2015) reportaron diferencias mayores al $50 \%$ en la corvina Cynoscion nebulosus. La separación por tallas efectuada a partir de los 26 días, aunque no forma parte del objetivo principal del trabajo, contribuyó en la fase final de cosecha y entrega de los peces a la granja; ya que permitió cosechar solo el tanque con la talla adecuada, así como, llevar el control de la cantidad disponible para entrega. 
La mayoría de las deformaciones esqueléticas genéticas en peces aparecen en las etapas de larvas y juveniles; son de considerable interés, debido a que reducen la sobrevivencia larvaria y el crecimiento de los peces en los laboratorios, y en las granjas de engorde los peces deformes deben ser descartados o vendidos a un menor precio (Gisbert, Fernández \& Estévez, 2008). El $6,43 \%$ de las deformidades esqueléticas observadas se consideran bajas en comparación con otros peces marinos (entre $15 \mathrm{y}$ $50 \%$ ) (Boglione et al., 2001); sin embargo, es posible que aumente, si se aplican metodologías de doble tinción para análisis esqueléticos que permiten transparentar los tejidos de las larvas y evidenciar malformaciones óseas en cráneo, columna y complejo caudal, entre otros (Gisbert, Alcaraz, Tovar-Ramírez \& Álvarez-González, 2014).

La principal deformidad presentada correspondió a una formación incompleta del opérculo (5,3\% del total de la población), que fue considerada por Moretti et al. (1999) como la menos perjudicial, e indica que una incidencia menor al $10 \%$ es aceptable. Esta deformidad se atribuye entre otros aspectos a deficiencias de vitamina C (Planas \& Cunha, 1999); no obstante, Gisbert et al. (2008) explican que es difícil determinar, en muchos casos, el origen de una malformación y que puede deberse a muchos factores.

En comparación con trabajos similares la sobrevivencia final promedio de los tres desoves 8,4\% a los 60 días, fue inferior la descrita por Álvarez-Lajonchére et al. (2012) en la misma cantidad de días de cultivo $(12,1 \pm 1,1 \%)$ y superior a la reportada por Boza-Abarca et al. (2008) (1,5\% - 171 días) y Sierrra-de-la-Rosa (2007) (1,8\% 44 dpe). El 8,4\% de sobrevivencia se considera adecuado para la producción preindustrial (piloto) de la especie.

\section{CONCLUSIÓN}

Costa Rica, con sus investigaciones en instituciones como el Parque Marino del Pacífico, ha logrado mejorar la biotecnología de producción de la especie. Se ha conseguido la reproducción de ejemplares adultos F2, se han aplicado metodologías que combinan sistemas de flujo abierto y recirculado (RAS), nuevas tecnologías de producción de alimento vivo como la implementación de los fotobirreactores y estrategias de manejo de los alevines por tallas.

Aunque este trabajo haya demostrado la factibilidad técnica de mejorar la producción de juveniles de esta especie; aún se requiere de mejoras en aspectos puntuales como nutrición de larvas en los primeros días de cultivo y mayor control microbiológico. Los resultados en sobrevivencia se consideran positivos.

\section{AGRADECIMIENTOS}

Se agradecen los aportes para el desarrollo de este trabajo al Dr. Ricardo Jiménez Montealegre y a la Escuela de Ciencias Biológicas de la Universidad Nacional; a Chen Chen Hui y al International Cooperation and Development Fund de la República de China (Taiwán); a Óscar Fajardo Espinoza y a Álvaro Otárola Fallas del Instituto Costarricense de Pesca y Acuicultura, y a la Asociación de Acuicultores de Paquera.

\section{DECLARACIÓN DE LA CONTRIBUCIÓN DE LOS AUTORES}

El porcentaje total de contribución para la conceptualización, preparación y corrección de este artículo fue el siguiente: $\mathrm{J}$. C. G. $50 \%$, M. C. O. $30 \%$ y A. H. U. $20 \%$. 


\section{DECLARACIÓN DE DISPONIBILIDAD DE LOS DATOS}

Los datos que respaldan los resultados de este estudio serán puestos a disposición por el autor correspondiente [J. C. G], previa solicitud razonable.

\section{REFERENCIAS}

Abdo-de la Parra, M. I., Rodríguez-Ibarra, L. E, Rodríguez-Montes de Oca, G., Velasco-Blanco, G., \& Ibarra-Casto, L. (2015). Estado actual del cultivo de larvas del pargo flamenco ( $\mathrm{Lu}$ tjanus guttatus). Latin American Journal of Aquatic Research, 43, 415-423. https://doi. org/10.3856/vol43-issue3-fulltext-3

Abdo-de-la-Parra, M. I., Rodríguez-Ibarra, L. E., Campillo-Martínez, F., Velasco-Blanco, G., García-Aguilar, N., Álvarez-Lajonchère, L., \& Voltolina, D. (2010). Efecto de la densidad de siembra sobre el crecimiento y supervivencia larval del pargo lunarejo Lutjanus guttatus. Revista de Biología Marina y Oceanografia, 45, 141-146. https://doi.org/10.4067/ S0718-19572010000100014

Álvarez-Lajonchére, L., Abdo-de-la-Parra, M. I., Rodríguez-Ibarra, L.,E., Velasco-Blanco,G., Puello-Cruz, A., González Rodríguez, B., Ibarra-Soto, A., \& Ibarra-Castro, L. (2012). The scale-up of spotted rose snapper, Lutjanus guttatus, larval rearing at Mazatlan, Mexico. Journal of World Aquaculture Society, 43, 411-422. https://doi. org/10.1111/j.1749-7345.2012.00573.x

Baras, E. \& Jobling, J. (2002). Dynamics of intracohort cannibalism in cultured fish. Aquaculture Researche, 33,461-479. https://doi. org/10.1046/j.1365-2109.2002.00732.x

Benetti, D.,D., Sardenberg, B., Welch, A., Hoenig, R., Orhun, M., \& Zink, I. (2008). Intensive larval husbandry and fingerling production of cobia Rachycentron canadum. Aquaculture, 281,(1-4),22-27. https://doi.org/10.1016/j. aquaculture.2008.03.030

Boglione, C., Gagliardia, F., Scardib, M., \& Cataudella, S. (2001). Skeletal descriptors and quality assessment in larvae and post-larvae of wild-caught and hatchery-reared gilthead sea bream (Sparus aurata L . 1758). Aquaculture, 192,1-22. https://doi.org/10.1016/ S0044-8486(00)00446-4

Boza-Abarca, J., Calvo-Vargas, E., Solis-Ortiz, N., \& Komen, J. (2008). Induced spawning and larval rearing of spotted rose snapper, Lutjanus guttatus, at the Marine Biology Station, Puntarenas, Costa Rica. Ciencias Marinas, 34, 239-252. https://doi.org/10.7773/ cm.v34i2.1246

Bromage, N., Jones, J., Randall, C., Thrush, M., Davies, B., Springate, J., Duston, J., \& Barker, G. (1992). Broodstock management, fecundity , egg quality and the timing of egg production in the rainbow trout (Oncarhynchus rnykiss). Aquaculture, 100, 141-166. https:// doi.org/10.1016/0044-8486(92)90355-o

Carvajal-Oses, M., Campos-Rodríguez, R., \& Herrera-Ulloa, A. (2016). Technical and economic feasibility of adding pure oxygen for rotifer production (Brachionus plicatilis) spotted snapper (Lutjanus guttatus) larvae feeding. Revista Tecnología en Marcha, 29(2),14-24. https://doi.org/10.18845/tm.v29i2.2688

Castillo-Vargasmachuca, S. G., Ponce-Pelafox, J., Arámbul-Muñoz, E., Rodríguez-Domínguez, G., \& Aragón-Noriega, E. (2016). The spotted rose snapper (Lutjanus guttatus, Steindachner 1869) farmed in marine cages: Review of growth models. Reviews in Aquaculture, 10, 376-384. https://doi.org/10.1111/raq.12166

Chacón-Guzmán, J., Carvajal-Oses, M., Agüero, P. T. \& Gatica, H. F. (2020). Comparison of fatty acids profile of the gonads and eggs of Lutjanus guttatus (Perciformes: Lutjanidae) obtained from wild and captive broodstock. Uniciencia, 34(1), 32-59. https://doi. org/10.15359/ru.34-1.3

Chacón-Guzmán, J., Carvajal-Oses, M., Herrera-Ulloa, A., \& Pauletto, S. (2019a). Concentración y tiempo máximo de exposición de juveniles de pargo manchado Lutjanus guttatus al eugenol Syzygium aromaticum. Revista Ciencias Marinas y Costeras, 11(1), 9-25. https://doi.org/10.15359/revmar.11-1.1

Chacón-Guzmán, J., Carvajal-Oses, M., Herrera-Ulloa, A., Corrales-Gómez, N., Granados-Cerdas, R., Otárola-Fallas, A., Marín-Alpizar, B., \& Fajardo-Espinoza, O. (2019b). Liberación de juveniles de pargo manchado Lutjanus guttatus (Steindachner, 1869), como 
estrategia para la conservación de la especie y educación ambiental marino costera. Gestión y Ambiente, 22(1). https://doi.org/https://doi. org/10.15446/ga.v22n1.76516

Di-Rienzo, J. A., Casanoves, F., Balzarini, M., González, L., Tablada, M., \& Robledo, Y. (2011). InfoStat versión 2011. Universidad Nacional de Córdoba.

Emata, A. C., Eullaran, B., \& Bagarinao, T. (1994). Induced spawning and early life description of the mangrove red snapper. Lutjanus argentimaculatus. Aquaculture, 121,381-387. https:// doi.org/10.1016/0044-8486(94)90272-0

Fischer, W., Krupp, F., Schneider, W., Sommer, C., Carpenter, K., \& Niem, V. (1995). Guía FAO para la identificación de especies para los fines de la pesca, Pacífico centro-oriental. FAO.

Froese, R. (2006). Cube law, condition factor and weight-length relationships: history, meta-analysis and recommendations. Journal of Applied Ichthyology, 22, 241-253. https://doi. org/10.1111/j.1439-0426.2006.00805.x

García-Ortega, A., Abdo-de-la-Parra, I., Duncan, N., Rodríguez-Ibarra, E., Velasco, G., González-Rodríguez, B., Puello-Cruz, A., \& Martínez, I. (2005). Larval rearing of spotted rose snapper Lutjanus guttatus under experimental conditions. In C.I. Hendry et al. (Eds.), Larvi 05 - Fish and shellfish larviculture symposium (pp. 172-175). European Aquaculture Society, Oostende.

García-Rojas, A., Vega-Bolaños, H., \& Quesada-Phillips, V. (2017). Dinámica anual de tintínidos en las aguas estuarinas de dos áreas marinas de pesca responsable en el Golfo. Uniciencia, 31(1),1-12. https://doi. org/10.15359/ru.31-1.1

Gisbert, E., Alcaraz, C., Tovar-Ramírez, D. \& Álvarez-González, C. (2014). Development of the axial skeleton in the bay snook Petenia splendida Gunther, 1862 (Perciformes: Cichlidae). Journal of Applied Ichthyology, 30, 783-789. https://doi.org/10.1111/jai.12512

Gisbert, E., Fernández, L., \& Estévez, A. (2008). Nutrición y morfogénesis: Efecto de la dieta sobre la calidad larvaria en peces. Universidad Autónoma de Nuevo León.

Guillard, R. (1975). Culture of phytoplankton for feeding marine invertebrates. In W. L. Smith, y M. H. Chanley (Eds.), Culture of Marine Invertebrate Animals (pp. 29-60).
Springer International Publishing. https://doi. org/10.1007/978-1-4615-8714-9 3

Gutiérrez-Sigeros, I., Ibarra-castro, L., \& Álvarez-Lajonchère, L. (2018). Natural spawning and scaling-up of yellowtail snapper (Ocyurus chrysurus): Larval rearing for the mass production of juveniles. Aquaculture, 491, 252-257. https://doi.org/10.1016/j. aquaculture.2018.03.048

Gutiérrez-Vargas, R., \& Durán-Delgado, M. (1998). Cultivo de pargo de la mancha Lutjanus guttatus (Pisces: Lutjanidae) en jaulas flotantes. Uniciencia, 15(1), 27-34.

Hamamoto, S., Kumagai, S., Nosaka, K., Manabe, S., Kasuga, A., \& Iwatsuki, Y. (1992). Reproductive behavior, eggs and larvae of a lutjanid fish, Lutjanus stellatus, observed in an aquarium. The Japanese Journal of Ichthyology, 39(3),219-228. https://doi.org/10.1007/ BF02905479

Hecht, T., \& Piennar, A. (1993). A review of cannibalism and its implications in in fish larviculture. Journal of the World Aquaculture Society, 24, 246-261. https://doi.or$\mathrm{g} / 10.1111 / \mathrm{j} .1749-7345.1993 . t b 00014 . \mathrm{x}$

Hernández, C., Sanchez-Gutierrez, Y., Hardy, R., Benitez-Hernández, A., Domínguez-Jiménez, P., González-Rodríguez, B., Osuna-Osuna, L., \& Tortoledo, O. (2014). The potential of pet-grade poultry by product meal to replace fish meal in the diet of the juvenile spotted rose snapper Lutjanus guttatus (Steindachner, 1869). Aquaculture of Nutrition, 20, 623-631. https://doi.org/10.1111/anu.12122

Hernández, C. H., Hernández, C., Martínez-Cordero, F., Castañeda-Lomas, N., Rodríguez-Domínguez, G., Tacon, A., \& Aragón-Noriega. (2016). Effect of density at harvest on the growth performance and profitability of hatchery-reared spotted rose snapper, Lutjanus guttatus, cultured in floating net cages. Journal of the World Aquaculture Society, 47, 5160. https://doi.org/10.1111/jwas.12253

Herrera-Ulloa, A., Chacón-Guzmán, J., Zúñiga-Calero, G., \& Jiménez-Montealegre, R. (2010). Spotted red snapper (Lutjanus guttatus) aquaculture research and development as socio-economic alternative for Costa Rican fishing communities. World Aquaculture, 41, 20-22.

Herrera-Ulloa, A., Chacón-Guzmán, J., Zúñiga-Calero, G., Fajardo-Espinoza, O., \& Jímenez-Montealegre, R. (2009). Acuicultura de 
pargo la mancha Lutjanus guttatus (Steindachner, 1869) en Costa Rica dentro de Oun enfoque ecosistémico. Revista de Ciencias Marinas y Costeras, 1, 197-213. https://doi. org/10.15359/revmar.1.11

Ibarra-Castro, L., \& Duncan, N. (2007). GnRHa-induced spawning of wild-caught spotted rose snapper Lutjanus guttatus. Aquaculture, 272, 737-746. https://doi.org/10.16/j. aquaculture.2007.09.007

Ibarra-Castro, L., Álvarez-Lajonchère, L., García-Aguilar, N., Abdo-de-la-Parra, M., \& Rodríguez-Ibarra, L. (2012). Generation cycle closure of the spotted rose snapper, Lutjanus guttatus, in captivity. Revista de Biología Marina y Oceanografia, 47, 333-337. https:// doi.org/10.4067/S0718-19572012000200015

Ibarra-Castro, L., Gutiérrez-Sigeros, I., Álvarez-Lajonchère, L., Durruty-Lagunes, C., \& Sánchez-Zamora, A. (2015). Desempeño reproductivo y primeros estadios de vida en corvina pinta Cynoscion nebulosus en cautiverio. Revista de Biología Marina y Oceanografia, 50, 439-451. https://doi.org/10.4067/ S0718-19572015000400004

Instituto Costarricense de Pesca y Acuicultura, \& Secretaría Ejecutiva de Planificación Sectorial Agropecuaria. (2019). Plan estratégico de la acuicultura en Costa Rica, 2019-2023. INCOPESCA. http://www.infoagro.go.cr/ documents/

Liao, I. C., Su, H., \& Chang, E. (2001). Techniques in finfish larviculture in Taiwan. Aquaculture, 200, 1-31. https://doi.org/10.1016/ S0044-8486(01)00692-5

Martínez-Cordero, F. J., Sanchez-Zazueta, E., \& Hernández, C. (2017). Investment analysis of marine cage culture by applying bioeconomic reference points: A case study of the spotted rose snapper (Lutjanus guttatus) in Mexico. Aquatic Economics Management, 22(2),219228. https://doi.org/10.1080/13657305.2017. 1295489

Mazón-Suástegui, J. M., Rosero-García, A., Avilés-Quevedo, A., Dumas, S., Vega, R., Rodríguez-Jaramillo, C., \& Tovar-Ramírez, D. (2016). Homeopathy for marine fish aquaculture: Increased growth and survival of juvenile spotted rose snapper Lutjanus guttatus. Homeopathy, 105, 32-33. https://doi. org/10.1016/j.homp.2015.12.055
Moretti, A., Fernández-Criado, M., Cittolin, G., \& Guidastri, R. (1999). Manual on hatchery production of seabass and gilthead seabream. FAO.

Naas, I., Naess, T., \& Harboe, T. (1992). Enhanced first feeding of halibut larvae (Hippoglossus hippoglossus L.) in green water. Aquaculture, 105, 143-156. https://doi. org/10.1016/0044-8486(92)90126-6

Ogle, J., \& Lotz, J. (2011). Characterization of an experimental indoor larval production system for red snapper. North American Journal of Aquaculture, 68, 86-91. https://doi. org/10.1577/A05-029.1

Palmer, P., Burke, M., Palmer, C., \& Burke, J. (2007). Developments in controlled green-water larval culture technologies for estuarine fishes in Queensland, Australia and elsewhere. Aquaculture, 272, 1-21. https://doi.org/ 10.1016/j. aquaculture.2007.06.018

Papanikos, N., Phelps, R., Williams, K., Ferry, A., $\&$ Maus, D. (2003). Egg and larval quality of natural and induced spawns of red snapper, Lutjanus campechanus. Fish Physiology and Biochemistry, 28, 487-488. https://doi. org/10.1023/B:FISH.0000030636.08228.4e

Peña, E., Hernández, C., Ibarra-Castro, L., \& Álvarez-González, C. (2017). In vitro protein digestibility of different grow- out stages of spotted rose snapper (Lutjanus guttatus, Steindachner, 1869). Aquaculture Nutrition, 23, 1204-1215. https://doi.org/ 10.1111/ anu. 12489

Planas, M., \& Cunha, I. (1999). Larviculture of marine fish : problems and perspectives. Aquaculture, 177, 171-190. https://doi.org/10.1016/ S0044-8486(99)00079-4

Qin, J., \& Fast, A. (1996). Size and feed dependent cannibalism with juvenile snakehead Channa striatus. Aquaculture, 313-320. https://doi. org/10.1016/0044-8486(96)01299-9 144

Rojo-Cebreros, A. H., Morales-Plascencia, M., Ibarra-Castro, L., Martínez-Brown, J., \& Medina-Jasso, M. (2016). Floculación de Nannochloropsis $s p$. inducida por hidróxido de sodio: eficiencia de floculación, efecto sobre la viabilidad microalgal y su uso como alimento para rotíferos. Latine American Joirnal Aquaculure Research, 44, 662-670. https://doi. org/10.3856/vol44-issue4-fulltext-1 
Rosenlund, G., Stoss, J., \& Talbot, C. (1997). Co-feeding marine fish larvae with inert and live diets. Aquaculture, 155, 183-191. https://doi. org/10.1016/S0044-8486(97)00116-6

Sierra-de-la-Rosa，J. (2007). Inducción hormonal (HCG) al desove y larvicultura del pargo lunarejo Lutjanus guttatus como alternativa de diversificación para la maricultura en el pacífico colombiano. Revista Electrónica en Ingeniería en Producción Acuícola, 3(3),47-60.

Silva, A., \& Castelló, J. (2005). Técnicas de producción de huevos y larvas de peces marinos. En A. Silva (Ed.), Cultivo de peces marinos (pp. 159-183). Universidad Católica del Norte Coquimbo.

Silva, A., \& Oliva, M. (2010). Review of the biology and cultivation of chilean flounder (Paralichthys adspersus). Latin American Journal of Aquatic Research, 38, 377-386. https://doi. org/10.3856/vol38-issue3-fulltext-3

Steindachner, F. (1869). Ichthyologische notizen. Sitzber Akad, 60(1), 290-318.

Suantika, G., Dhert, P., Nurhudah, M., \& Sorgeloos, P. (2000). High-density production of the rotifer Brachionus plicatilis in a recirculation system: consideration of water quality, zootechnical and nutritional aspects. Aquacultural Engineering, 21(3), 201-214. https://doi. org/10.1016/S0144-8609(99)00031-X
Tovar-Ramírez, D., Tales, J. S., Salas-Leiva, A., Hernández-Contreras, G. G., Asencio-Alcudia, J., Le-Du, M., Burgoin-Cota, C. A., Álvarez-González, R., Llera-Herrera, E., Gisbert, I., Fernández, J. C., Pérez-Urbiola, L., Ibarra-Castro, J. M., Mazón-Suástegui, E. J., Núñez-Vázquez, L. T., \& Reyes-Becerril, M. (2017). Enfoques transcriptómicos en el jurel Seriola rivoliana. En L. Cruz-Suárez et al. (Eds.), Investigación y desarrollo en nutrición acuícola (pp. 389-406). Universidad Autónoma de Nuevo León.

Uribe, E., \& Rangel, J. (2004). Curso Internacional de acuicultura de peces marinos. Fotobiorreactor tubular (pp. 115-125). En A. Silva (Ed.). Universidad Católica del Norte.

Velarde, D., Lara, C., Durán, M., Barlett, F., Sardenberg, B., \& Benetti, D. (2012). Acuacultura integrada del pargo roja establecida en América Central. Global Aquaculture Advocate, $65,8-9$.

\section{(c) (i) $(9)$}

Optimización del cultivo larvario para la producción de juveniles del pargo manchado Lutjanus guttatus en Costa Rica (Jonathan Chacón-Guzmán • Milagro Carvajal-Oses • Ángel Herrera-Ulloa) Uniciencia is protected by Attribution-NonCommercial-NoDerivs 3.0 Unported (CC BY-NC-ND 3.0) 